\title{
Measuring the Bid-Ask Spread: A Note on the Potential Downward Bias of the Thompson-Waller Estimator
}

\author{
Yoichi Otsubo* \\ Luxembourg School of Finance \\ University of Luxembourg
}

February 2013

\begin{abstract}
The upward bias of the widely used Thompson-Waller estimator has been pointed out in the literature. In contrast the current paper provides a case the estimator would have downward bias. Such case satisfies the two conditions: (i) the buy (sell) order tends to follow buy (sell) order and (ii) the price change associated to such orders are small. The upward bias might be cancelled out by downward bias, and the estimator might perform better than the other methods such as Wang-Yau-Baptiste used by the CFTC. The high-frequency data of the emissions market allows us to provide an empirical evidence.
\end{abstract}

Keywords: Bid-ask spread; Futures market; Market microstructure; Thompson-Waller estimator

JEL Classification: C18; G12; G19

\footnotetext{
* University of Luxembourg, Luxembourg School of Finance, 4 rue Albert Borschette, L-1246 Luxembourg, email: yoichi.otsubo@uni.lu, +352-466644-6836.
} 


\section{Introduction}

The bid-ask spread is one of the important measures of market liquidity. Narrower spreads facilitate trades and lower transaction costs. The main difficulty in estimating spreads in futures market is that the information on quotes are usually not provided. A number of approaches have been taken to estimate spreads.

One of the popular methods to estimate the spread is the Thompson-Waller (TW) estimator. Applications of the measure are found in Thompson and Waller (1988), Thompson, Eales and Seibold (1988), Ma, Peterson, and Sears (1992), and Bryant and Haigh (2004) for example. The TW estimate equates to the average bid-ask spread if the expected true price change and the variance of true price change are both zero. Under the violation of this condition, it consists of two components, the bid-ask spread and the magnitude of true price changes, and it would be biased upward. ${ }^{1}$ While the upward bias due to the change in the true price has been pointed out in the literature, the potential downward bias has not been carefully discussed.

This study provide a case that the TW estimator would have downward bias. Such case is that (i) the buy (sell) order tends to follow buy (sell) order, and (ii) the price change associated to such orders are small. Furthermore, the upward bias of the TW estimator would be canceled out by the downward bias, and in such case the estimator would perform better than the other absolute price change methods such as the Wang-Yau-Baptiste (1997) used by the U.S. Commodity Futures Trading Commission (CFTC) which suppose to modify the TW estimator.

The empirical finding from the application to the European Union Allowances (EUA) futures contract trading implies that its trading pattern and the price change provide the conditions that reduce the bias of the TW estimator. The TW estimates are not remarkably different to the estimates provided by the trade indicator model approach of Roll (1984) with ordinary least squares estimation.

The remaining part of this paper is organized as follows. Section 2 describes the spread estimation methods and discuss the possible bias of the estimators. Section 3 discusses the bias of the spread estimators from the empirical findings. Finally, section 4 summarizes our findings.

\footnotetext{
${ }_{1}$ This upward bias in the TW estimator has been pointed out in the literature, Smith and Whaley (1994) for example.
} 


\section{Spread Estimation Methods}

This section presents methodologies for bid-ask spread estimation. These methods considered in the present study can be categorized in two types: the absolute price change measures and the trade indicator model parameter estimation.

\subsection{Absolute Price Change}

I start with describing the three absolute price change measures. All of the methods are variants of average absolute change in the transaction price. The potential bias of the estimators are discussed at the last subsection.

\subsubsection{Thompson-Waller}

The idea of measuring the bid-ask spread by the average of absolute price change is first applied by Thompson and Waller (1987). The change in transaction price, $\Delta p_{t}$, can be expressed as

$$
\Delta p_{t}=\frac{S}{2} I_{t}+\Delta m_{t}
$$

where $S$ is the spread, $\Delta m_{t}$ is the change in the true price, and $I_{t}$ is the indicator variable, $I_{t}=2$ if a buy order follows a sell order, $I_{t}=-2$ if a sell order follows a buy order, and $I_{t}=0$ otherwise. Then the TW spread estimate is given by,

$$
S^{T W}=\sum_{t^{+}=1}^{T^{+}}\left|\Delta p_{t^{+}}\right|^{+} / T^{+} .
$$

$T^{+}$is the number of non-zero changes in the transactions prices.

\subsubsection{Modified Thompson-Waller}

If the trade initiation of the executed transaction, $I_{t}$, are observable, the TW estimator can be modified as

$$
S^{M T W}=\sum_{t^{\prime}=1}^{T^{\prime}}\left|\Delta p_{t^{\prime}}\right| / T^{\prime}
$$

where $\Delta p_{i^{\prime}}$ is the price change that moves from bid to ask (or ask to bid) and $T^{\prime}$ is the number of such changes in the transactions prices.

The estimates $S^{T W}$ and $S^{M T W}$ equate to the average bid-ask spread if the expected true price change and the variance of true price change are both zero. Under the violation of this condition, as we can see from (1), they consist of two components, the bid-ask spread and the magnitude of true price changes, and it would be biased upward. 


\subsubsection{Wang-Yau-Baptiste}

Wang, Yau, and Baptiste (1997) attempt to reduce the bias of the TW estimator by discarding any price change that follows another price change of the same sign. ${ }^{2}$ The method is used by the CFTC to estimate the actual bid-ask spread. The Wang-Yau-Baptiste estimator is given as

$$
S^{W Y B}=\sum_{t^{\prime \prime}=1}^{T^{\prime \prime}}\left|\Delta p_{t^{\prime \prime}}\right| / T^{\prime \prime}
$$

where $\Delta p_{t^{\prime \prime}}$ is the price change that moves in a different direction from the previous change and $T^{\prime \prime}$ is the number of such changes in the transactions prices.

\subsubsection{Bias of the Absolute Price Change Estimators}

If the assumptions of the true price changes are violated, $S^{T W}$ and $S^{M T W}$ would have upward bias. While the literature has pointed out this bias due to the true price changes, the possibility of the TW estimator having downward bias has not been carefully discussed.

Suppose that a buyer initiated trade follows a buyer initiated trade. In such case, the absolute price change captured by $S^{T W}$ is not the spread, but it is the change in the best ask price. If the two consecutive trades are executed in a short period, it is likely that the change in the lowest ask price is small. When these changes are smaller than the average spread, $S^{T W}$ would be biased downward.

It is most likely that the unrealistic assumptions of the true price changes are violated and therefore TW estimator suffers from upward bias. However, if a market observes many trades in such a manner discussed above, the upward bias caused by the variance of the true price changes would be canceled out by the downward bias.

To calculate $S^{M T W}$, change in the price between two consecutive buyer (seller) initiated trades are discarded. Thus, the price changes which potentially offset the upward bias of the estimator are filtered out. The modified TW estimator is, because of the modification, would have greater bias than $S^{T W}$.

The WYB estimator discards the price changes that followed by another change with same direction. It is likely that a positive (negative) price change is caused by the placement of a buy (sell) order. Hence, the price changes discarded to compute $S^{W Y B}$ would be similar to those discarded to calculate $S^{M T W}$. Thus, although the WYB estimator discards such price changes in

\footnotetext{
${ }^{2}$ This estimator was applied in Bryant and Haigh (2004), Tse and Zabotina (2004) and Chou and Chung (2005) for example.
} 
order to reduce the bias, it would have greater bias than $S^{T W}$.

Therefore, although the assumptions which TW estimator is based on are unrealistic and would have bias, it would perform better than the other absolute price change estimators in practice. Applying those methods to the intra day data of European emissions market, further discussions are done with the empirical findings in later section.

\section{$2.2 \quad$ Trade Indicator Model}

In this section,the Roll model (1984) of bid, ask and transaction prices are summarized. The model describe the trade direction by trade indicator variables.

\subsubsection{Roll}

Roll (1984) assumes an Informationally efficient market, and assumes that the true price $m_{t}$ follows a random walk process,

$$
m_{t}=m_{t-1}+u_{t} .
$$

where the $u_{t}$ are i.i.d. zero-mean random variables with variance $\sigma_{u}^{2}$. In a competitive market, traders will set the bid $p_{t}^{b}$ and ask $p_{t}^{a}$ quotes wide enough to cover their execution cost, $c$. Namely,

$$
\begin{aligned}
& p_{t}^{b}=m_{t}-c, \\
& p_{t}^{a}=m_{t}+c .
\end{aligned}
$$

The bid-ask spread is $p_{t}^{a}-p_{t}^{b}=2 c$, and $c$ can be interpreted as the half-spread. Denoting the trade direction by $x_{t}$, the transaction price $p_{t}$ can be represented as,

$$
p_{t}=\left\{\begin{array}{lll}
p_{t}^{b} & \text { if } & x_{t}=-1 \\
p_{t}^{a} & \text { if } & x_{t}=+1
\end{array}\right.
$$

where trade direction of the incoming order is given by the Bernoulli random variable $x_{t} \in$ $\{-1,+1\}$. $\quad-1$ indicates a sell order and +1 indicates a buy order. Orders are assumed to arrive with equal probability, serially independent. It is also assumed that the trade direction arrival is independent of the efficient price innovation $u_{t}$.

The Roll model has two parameters, $c$ and $\sigma_{u}^{2}$. These are estimated from the variance and first-order autocovariance of the price changes, $\Delta p_{t}$. The variance is

$$
\begin{aligned}
\operatorname{Var}\left(\Delta p_{t}\right) & =E\left[\left(\Delta p_{t}\right)^{2}\right] \\
& =E\left[x_{t-1}^{2} c^{2}+x_{t}^{2} c^{2}-2 x_{t-1} x_{t} c^{2}-2 x_{t-1} u_{t} c+2 x_{t} u_{t} c+u_{t}^{2}\right] \\
& =2 c^{2}+\sigma_{u}^{2} .
\end{aligned}
$$


The last equality follows because in expectation, all of the cross-products vanish except for those involving $x_{t}^{2}, x_{t-1}^{2}$, and $u_{t}^{2}$. The first order covariance is

$$
\begin{aligned}
\operatorname{Cov}\left(\Delta p_{t}, \Delta p_{t-1}\right)= & E\left[\Delta p_{t-1} \Delta p_{t}\right] \\
= & E\left[c^{2}\left(x_{t-2} x_{t-1}-x_{t-1}^{2}-x_{t-2} x_{t}+x_{t-1} x_{t}\right)\right. \\
& \left.+c\left(x_{t} u_{t-1}-x_{t-1} u_{t-1}+u_{t} x_{t-1}-u_{t} x_{t-2}\right)\right] \\
= & -c^{2} .
\end{aligned}
$$

It is easily verified that all autocovariances of order 2 or higher are zero. From the above, the spread, $S^{\text {Roll }}$, is estimated as

$$
S_{t}^{\text {Roll }}=2 \sqrt{-\operatorname{Cov}\left(\Delta p_{t}, \Delta p_{t-1}\right)}
$$

and $\sigma_{u}^{2}=\operatorname{Var}\left(\Delta p_{t}\right)+2 \operatorname{Cov}\left(\Delta p_{t}, \Delta p_{t-1}\right)$.

\subsubsection{Roll OLS}

From (5) to (7), the transaction price process is,

$$
\Delta p_{t}=m_{t}+c x_{t}-\left(m_{t-1}+c x_{t-1}\right)=c \Delta x_{t}+u_{t} .
$$

If one can observe the trade initiations, the above model can be estimated by usual ordinary least squares (OLS) regression. Hence the Roll OLS spread estimate is

$$
S_{t}^{R O L S}=2 \widehat{c}
$$

where $\widehat{c}$ is the OLS estimate of (11). Note that the OLS does not require the trade initiation variable to be serially uncorrelated.

\section{Empirical Analysis: Application to the Emissions Market Data}

The largest market for carbon trading is the European Union Emissions Trading System (EU ETS), a cap and trade scheme that emerged out of the Kyoto Protocol. EUA is traded on the European Climate Exchange (ECX). The ECX has, since the start of carbon exchange trading in 2005, been the leading venue. In 2009, the ECX processed $65.6 \%$ of the screen based trading volume in EUA.

The intra-day obsrvation used here are transaction prices from the ECX for the December 2009 futures contract of EUA. The data contains a record of each trade price and trade direction (whether the trade falls on the best bid or ask). The sample begins on January 2, 2009 and ends on 
December 14, 2009 (244 trading days). All of the observations are used to compute the estimates.

Firstly the bid-ask spreads of the market are estimated by the absolute price change methods. The empirical findings imply that the EUA futures market would provide the conditions which reduces the bias of the TW estimator. Figure 1 plots the TW, the modified TW and the WYB spread estimates.

[Insert Figure 1: ECX 2009 EUA Futures Monthly Spreads: TW, Modified TW, and WYB Estimates]

All the spread estimates tend to narrow gradually through time. The TW, WYB and modified TW spread decrease 42\%,47\% and 50\% between January and December, respectively.

The yearly average spreads computed by three methods are $€ 0.0221, € 0.0258$ and $€ 0.0287$. The spread estimate of the first six months varies greatly by method. In particular, the modified TW spreads are $€ 0.0075$ to $€ 0.0110$ wider than the TW spreads.

The WYB and modified TW spreads are consistently wider than the TW spread. This result is expected from our discussion in section 2. The TW estimates seem to be less upward biased. The continuous execution of orders in the same side, which is discarded to calculate $S^{M T W}$ and $S^{W Y B}$ would reduce the bias of $S^{M T W}$. Table 1 reports the number of such orders.

[Insert Table 1: Number of Observations used in the Spread Estimation: TW, Modified TW, and WYB Estimates]

On average, $65 \%$ of the observations used in the TW estimation are the orders continuously traded in the same side. If the price change of such continuous orders are significantly smaller than the price change of the other cases, TW estimates are considered to have downward bias, which would offset the upward bias due to the change in the true price. Figure 2 compares the monthly average of the absolute price change of the orders discarded to calculate $S^{M T W}$, and $S^{M T W}$ themselves.

[Insert Figure 2: The Monthly Average of the Discarded Absolute Price Change and the Modified TW Estimates]

The size of price changes from buyer (seller) initiated to buyer (seller) initiated are remarkably smaller than the other case. As we have seen above, notable size of the observations used in the TW estimation are such small price changes. The wider spread of WYB and the modified TW 
spreads would be explained by the elimination of those small price changes. Thus, the gap between the modified TW and the TW spreads would be considered as the magnitude of the downward bias of TW estimates.

Although the modified TW spreads would not suffer from the downward bias, it would suffer from the upward bias due to the change in the true price. Prior to get into that discussion, we examine the results of the Roll spread estimates. Figure 3 plots the Roll covariance estimates and the Roll OLS estimates.

[Insert Figure 3: ECX 2009 EUA Futures Monthly Spreads: The Roll Covariance and the Roll OLS Estimates]

The Roll covariance estimates and the Roll OLS estimates for the first four months are remarkably different. The Roll model assumes that the trade initiations are serially uncorrelated and are also uncorrelated with changes in the efficient price. We already confirmed that the first assumption is not appropriate with our data, from the observation that the $65 \%$ of the order is followed by the same side of the order.

The Roll OLS estimation does not require the serial uncorrelation of the trade directions. Hence, we expect that the Roll OLS estimate would be a better estimate than the Roll covariance estimates. Furthermore, by analyzing the Roll OLS residuals, $\widehat{u}_{t}$, we examine the factor of upward bias in the absolute price change methods. Figure 4 reports the modified TW spread, the Roll OLS and the standard deviation of its residuals.

[Insert Figure 4: ECX 2009 EUA Futures Monthly Spreads and the Estimated Size of the True Price Change: The Modified Thompson-Waller and the Roll OLS Estimates]

The standard deviation of the Roll OLS residuals, $\sqrt{\operatorname{Var}\left(\widehat{u}_{t}\right)}$ is considered as an ad-hoc estimates of the size of the change in the true price. The figure indicates that the larger the variances of the changes in the true price, the greater the gap between the estimates. That would be an empirical evidence of the upward bias in the modified TW estimate caused by the magnitude of the true price change.

All the three estimates, $S^{T W}, S^{M T W}$ and $S^{W Y B}$ would be upward biased due to the true price change. Our discussion in section 2 provide a possible case that the upward bias of $S^{T W}$ would be canceled out, while the bias of $S^{M T W}$ and $S^{W Y B}$ would not. The findings in the pattern of the order arrivals, the change in transaction price, and the change in the true price suggest that 
the ECX is such a case. Thus, although the assumptions for the TW estimator are obviously violated, since buy (sell) order tends to follow buy (sell) order and the corresponding price change are relatively small in the ECX, the TW estimator would provide better estimate than the other two absolute price change estimators for this particular market.

\section{Conclusion}

This study provides a case that the TW estimator would have downward bias. Such case is that (i) the buy (sell) order tends to follow buy (sell) order and (ii) the price change associated to such orders are small. While the upward bias due to the change in the true price has been pointed out in the literature, the downward bias has not been carefully discussed. Furthermore, the upward bias of the TW estimator would be canceled out by the downward bias, and in such case the estimator would perform better than the other absolute price change methods such as WYB estimator, used by the CFTC, which suppose to modify the TW estimator.

The empirical finding from the application to the EUA futures contract trading implies that its trading pattern and the price change provide the conditions that reduce the bias of the TW estimator. The TW estimates are not remarkably different to the estimates provided by the trade indicator model approach as Roll OLS. 


\section{References}

Bryant, Henry and Michael Haigh (2004)."Bid-Ask Spreads in Commodity Futures Markets," Applied Financial Economics 14, 923-936.

Chou, Robin and Huimin Chung (2005). "Decimalization, Trading Costs, and Information Transmission between ETFs and Index Futures," The Journal of Futures Markets 26, 131-151.

Ma, Christopher K., Richard L. Peterson, and Stephen R. Sears (1992). "Trading noise, adverse selection, and intraday bid-ask spreads in futures markets," The Journal of Futures Markets 12, $519-538$.

Roll, Richard (1984). "A Simple Implicit Measure of the Effective Bid-ask Spread in an Efficient Market," Journal of Finance 23, 1127-1139.

Smith, Tom and Robert E. Whaley (1994). "Estimating the Effective Bid/Ask Spread from Time and Sales Data," The Journal of Futures Markets 14, 437-455.

Thompson, Sarahelen, James S. Eales, and David Seibold (1993). "Comparison of liquidity costs between the Kansas City and Chicago wheat futures contracts," Journal of Agriculture and Resource Economics 18, 185-197.

Thompson, Sarahelen and Mark Waller (1988). "Determinants of Liquidity Costs in Commodity Futures Markets," Review of Futures Markets 7, 110-126.

Thompson, Sarahelen and Mark Waller (1987). "The Execution Cost of Trading in Commodity Futures Markets," Food Research Institute Studies 20, 141-163.

Wang, George H. K., Jot Yau, and Tony Baptiste (1997). "Trading volume and transaction costs in futures markets," The Journal of Futures Markets 17, 757-780.

Tse, Yuman and Tatyana Zabotina (2004). "Do Designated Market Makers Improve Liquidity in Open-Outcry Futures Markets?," The Journal of Futures Markets 24, 479-502. 
Table 1

Number of Observations used in the Spread Estimation: Thompson-Waller, Modified Thompson-Waller, and Wang-Yau-Baptiste Estimates

\begin{tabular}{lcccc}
\hline Month & $T^{+}$ & $T^{\prime}$ & $T^{\prime \prime}$ & $T^{+}-T^{\prime}$ \\
\hline \hline January & 7073 & 2614 & 2784 & 4459 \\
February & 8746 & 3270 & 3227 & 5476 \\
March & 10483 & 3752 & 3864 & 6731 \\
April & 14988 & 5055 & 5825 & 9933 \\
May & 11658 & 3672 & 4347 & 7986 \\
June & 13306 & 4480 & 5106 & 8826 \\
July & 10186 & 3374 & 3507 & 6812 \\
August & 8051 & 2685 & 2937 & 5366 \\
September & 6493 & 2213 & 2061 & 4280 \\
October & 6163 & 2083 & 1877 & 4080 \\
November & 5004 & 1831 & 1333 & 3173 \\
December & 1516 & 618 & 408 & 898 \\
\hline
\end{tabular}

$T^{+}, T^{\prime}$ and $T^{\prime \prime}$ are the number of observations used in estimating the the Thompson-Waller estimator, (2), the modified Thompson-Waller estimator, (3), and the Wang-Yau-Baptiste estimator, (4), respectively. $T^{+}-T^{\prime}$ is the number of continuous arrivals of orders in the same side. 
Figure 1

ECX 2009 EUA Futures Monthly Spreads: Thompson-Waller, Modified Thompson-Waller, and Wang-Yau-Baptiste Estimates

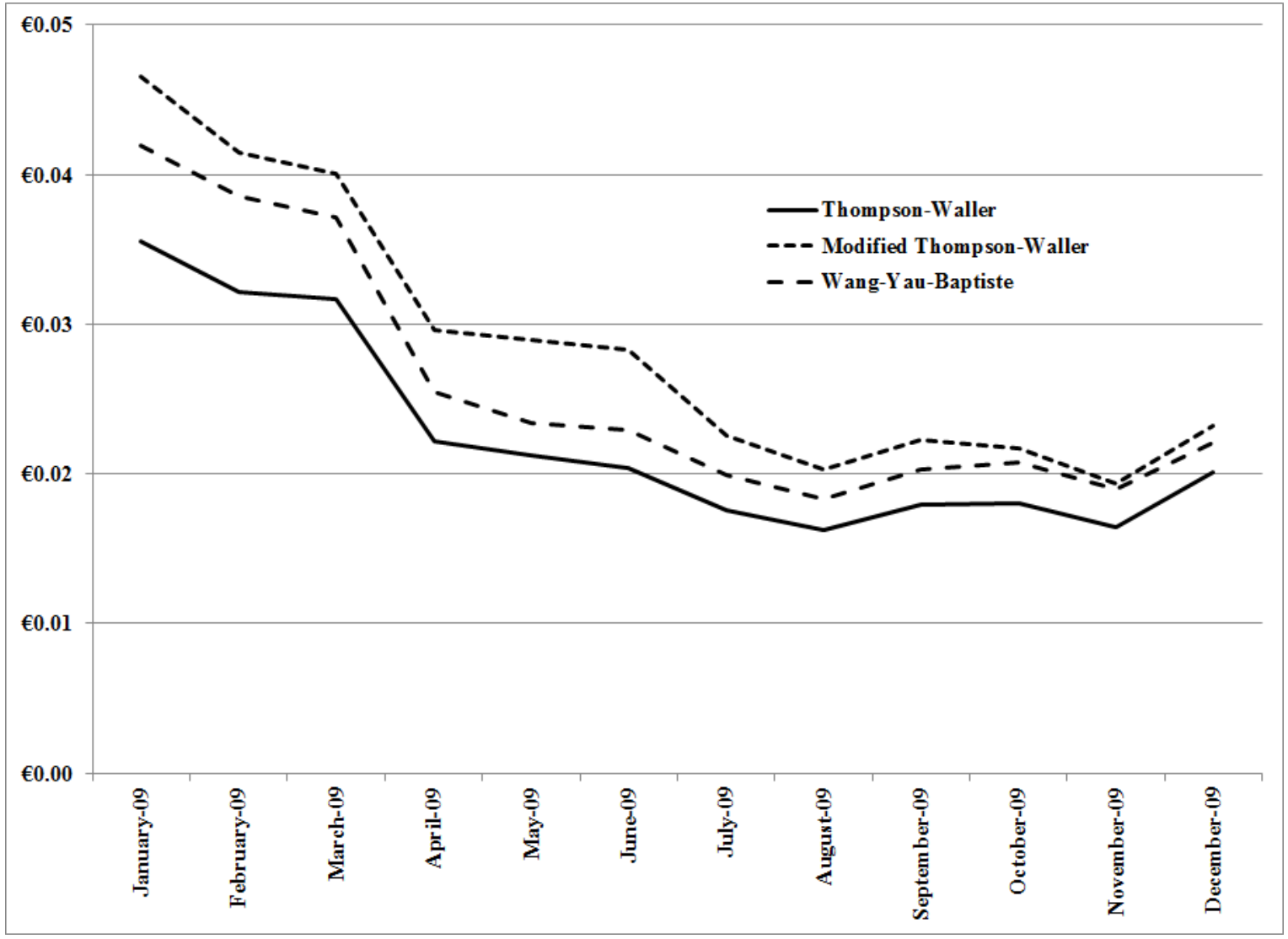

The figure displays monthly average bid-ask spread estimates of EUA December expiry futures from the European Climate Exchange. Estimates are computed using the Thompson-Waller estimator, (2), the modified TW estimator, (3) and the Wang-Yau-Baptiste estimator (4). 
Figure 2

The Monthly Average of the Discarded Absolute Price Change and the Modified Thompson-Waller Estimates

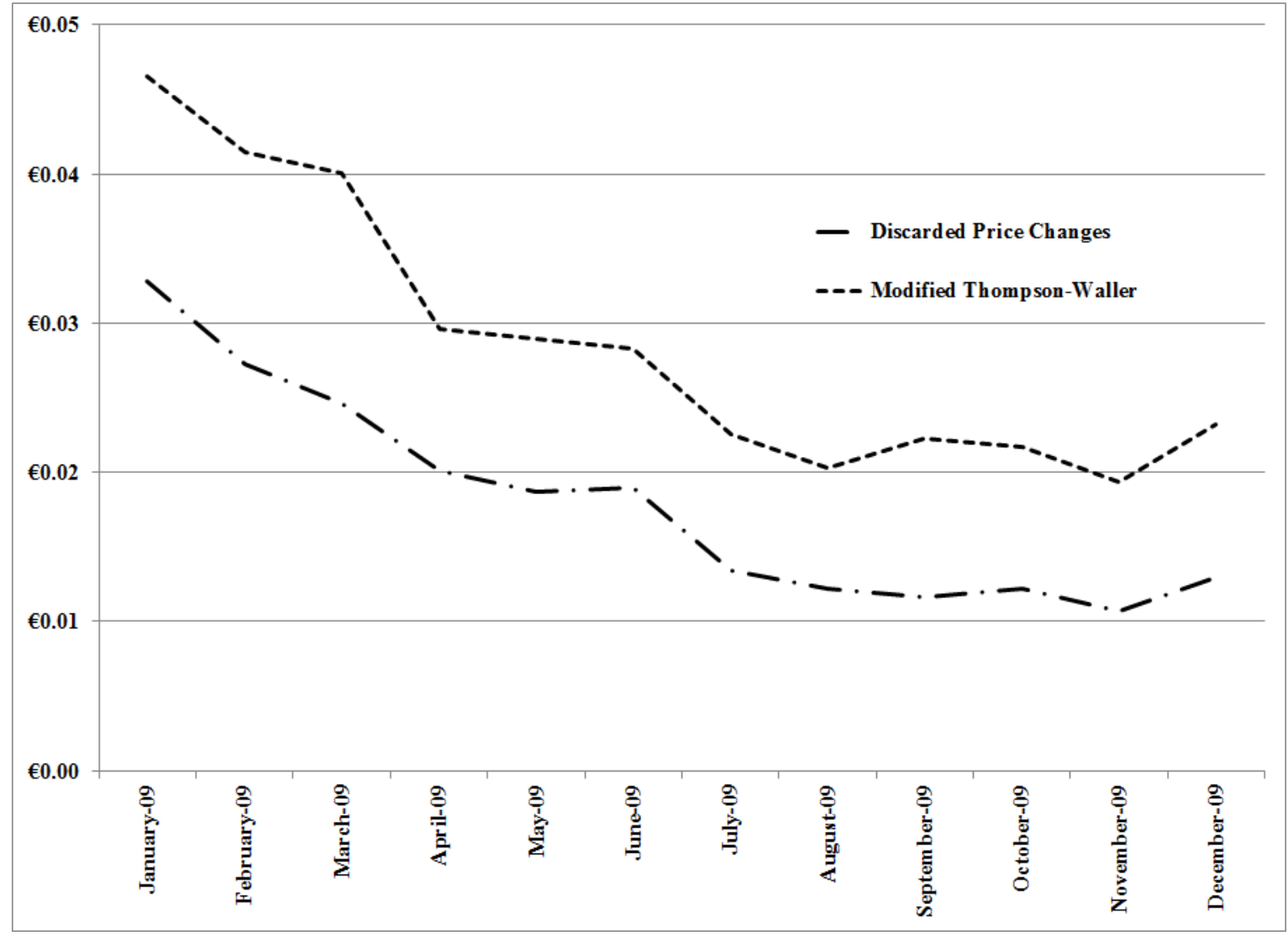

The figure displays the monthly average of the discarded absolute price changes to compute the modified Thompson-Waller estimates, and the modified Thompson-Waller estimates, (3). 
Figure 3

ECX 2009 EUA Futures Monthly Spreads: The Roll Covariance and the Roll OLS Estimates

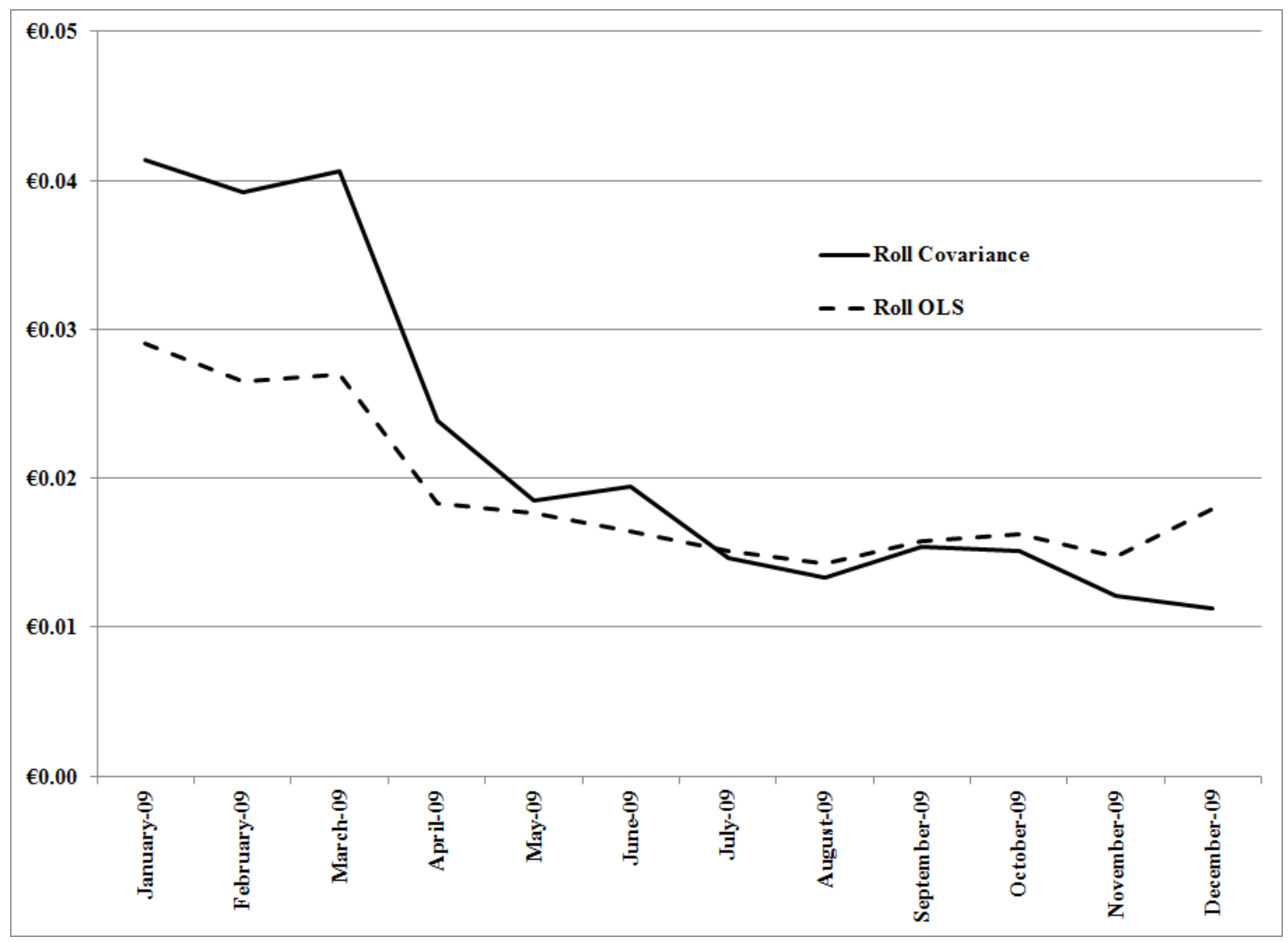

The figure displays monthly average bid-ask spread estimates of EUA December expiry futures from the European Climate Exchange. Estimates are computed using the Roll covariance estimator, (10), and the Roll OLS estimator, (12). 
Figure 4

ECX 2009 EUA Futures Monthly Spreads: The Modified Thompson Waller and the Roll OLS Estimates

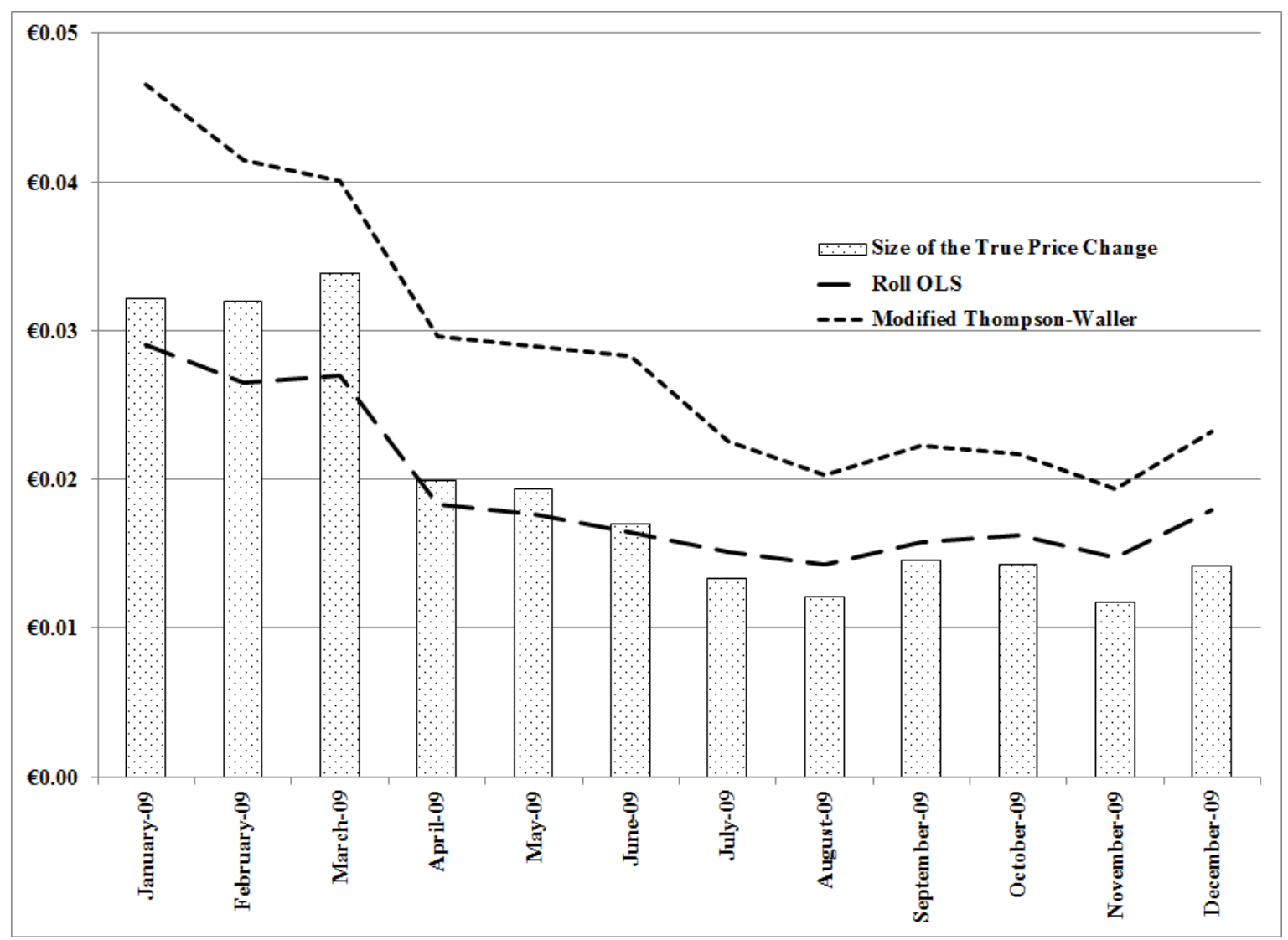

The figure displays monthly average bid-ask spread estimates of EUA December expiry futures from the European Climate Exchange. Estimates are computed using the modified ThompsonWaller estimator, (3), and the Roll OLS estimator, (12). The monthly average size of the true price change are the standard deviation of the Roll OLS residuals. 